\title{
Cancer immunotherapy: present scenarios and the future of immunotherapy
}

\author{
Dwaipayan Chakraborty $^{1}$. Subhadip Pati ${ }^{1} \cdot$ Sayantan Bose ${ }^{1} \cdot$ Subhanki Dhar $^{1} \cdot$ Saikat Dutta $^{1} \cdot$ Gaurisankar Sa $^{1}$
}

Received: 9 November 2018 / Accepted: 12 April 2019 / Published online: 18 May 2019

(c) Archana Sharma Foundation of Calcutta 2019

\begin{abstract}
One of the major emerging modes of treatment for cancer patients in the current healthcare systems has been immunotherapy. Various approaches of immunotherapy have been proved to be useful against tumor progression in laboratory settings. Few have also been showing promising results in clinical trials. However, many clinical trials have also pointed out the adverse side effects of immunotherapy on tumor patients. In this context, we discuss about the success and failures of the present modes of immunotherapy available to patients and also about the emerging target points that are coming up and may be crucial in the success of future immunotherapy. The upcoming researches involving new players in the immunotherapy arena like Breg and Treg and how combinatorial therapies will be needed to counter the side effects that are exhibited by the present techniques of immunotherapy have been discussed in the review. This review will provide a broad picture of the current scenario of cutting edge immunotherapy and the newer methods that can be utilized to prevent the failures and ensure success in future clinical trials involving immunotherapy.
\end{abstract}

Keywords Cancer immunotherapy $\cdot \mathrm{T}$ regulatory cell $\cdot \mathrm{B}$ regulatory cell $\cdot \mathrm{CAR} \mathrm{T}$ cells $\cdot$ Immunoediting

\section{Introduction}

Immunotherapy has emerged to be a highly successful strategy for treating malignancies in the last decade. Advances in understanding of the dynamic interaction between the immune system and cancer have resulted in the development of a number of novel therapeutic strategies that harness several aspects of the immune system to treat cancer. Although vague ideas about the association of immune system with tumor development existed for over a century, concrete developments in cancer immunotherapy began after the well accepted immunoediting hypothesis put forward by Robert Schreiber in 2003 [21]. The basic mechanism of

This article is based on the presentation made during 18th All India Congress of Cytology and Genetics and International Symposium on "Translating Genes and Genomes" held at CSIRIndian Institute of Chemical Biology, Kolkata in collaboration with Archana Sharma Foundation of Calcutta during January 29-31, 2018.

Gaurisankar Sa

gauri@jcbose.ac.in

1 Division of Molecular Medicine, Bose Institute, P-1/12, CIT Scheme VII M, Kolkata 700054, India anti-tumor immune response depends on efficient capture and presentation of tumor antigens by antigen presenting cells like dendritic cells. Proper immunogenic stimulus during antigen capture leads to maturation of dendritic cells to efficiently induce a $\mathrm{T}$ cell response by interaction of CD28 with CD80/86. However, generation of such a proper immune response is often hindered by a number of factors. Improper maturation signals to dendritic cells often lead to induction of tolerogenic response resulting in T-cell anergy and generation of immunosuppressive Treg cells. Thus current immunotherapy strategies tend to target this process at various stages like promotion of antigen presentation by dendritic cells, enhancing T-cell response against tumor and reduction of immunosuppressive factors in the tumor microenvironment [56]. By far the greatest success of cancer immunotherapy has been through the use of monoclonal antibodies against CTLA4 and PD-1. CTLA4 has been showed to be an inhibitory molecule that can dampen normal T-cell response [42, 90]. Anti-CTLA4 antibodies were shown to be highly effective in reducing murine tumors, which paved the way for their therapeutic use [46]. In 2011, the U.S Food and Drug Administration (FDA) approved the use of anti-CTLA4 antibodies (Ipilimumab) for clinical use and since then it has shown promising effects especially 
in melanoma patients [68]. Another checkpoint inhibition therapy that has achieved clinical success is the blockage of PD1-PDL1 axis. Programmed death ligand-1 (PD1) is also an inhibitory molecule present on immune cells which upon interaction with its ligand (PDL1) could dampen the immune response. The use of antibodies that block the PD1 pathway have also shown remarkable success in clinical trials [20, 91]. Apart from checkpoint inhibition another impressive line of immunotherapy has been the use of genetically engineered T-cells, known as Chimeric Antigen Receptor (CAR) T-cells that have enhanced ability to recognize tumour antigens. CAR-T cells have shown promising effects in B-cell malignancies [66]. However if the total long-term survival rate is considered, these therapies have only shown modest success. One of the major reasons of the failure of immunotherapy is the presence of immunosuppressive network in the tumor microenvironment [88]. Treg cells have gained importance in this regard and are considered to be a major mechanism by which tumors induce peripheral tolerance and escape from immunosurveillance. Depletion of Tregs can lead to rejuvenation of the immune system so that it can mount an effective response against the tumor and this can also complement the existent immunotherapeutic strategies [17]. However these therapies alone can only achieve limited success against the vast variety of malignancies that have different immunogenic potentials. Hence the focus has shifted towards the formulation of rational combinations of different immunotherapeutic agents to target multiple immunological pathways and aim towards restoration of an immune homeostasis to successfully mount a proper immunological response against cancer [62]. In this review, we summarise the progress of immunotherapy till now, the general strategies involved and the emerging therapies especially focusing on Treg based therapies. Moreover we also try to outline the concerns regarding the efficacy and safety of immunotherapies and the possible combinatorial approaches that might be utilized in the future (Fig. 1).

\section{Current immunotherapy procedures using T-cell}

Tumor cells generally express antigens of two types, one is tumor-specific antigens (solely expressed by tumor cells) e.g., mutated product of Ras, p53, Bcr/Abl and tumor associated antigens (also expressed by normal cells) whose expression is aberrant or deregulated in tumors e.g., carcinoembryonic antigen (CEA), cancer testis antigen (CTA). The number of CTLs (CD8+ Cytotoxic T lymphocyte) which are tumor antigen specific is low and the tumor microenvironment is immunosuppressive [86]. Therefore the adoptive transfer method is used. The concept of adoptive transfer of CTLs is isolation of tumor infiltrating lymphocytes (TILs), those are anti-tumorigenic in nature and culture the cells with proper activation signals (IL-2 and anti-CD3 antibody) and co-culturing with APC which present tumor antigen in

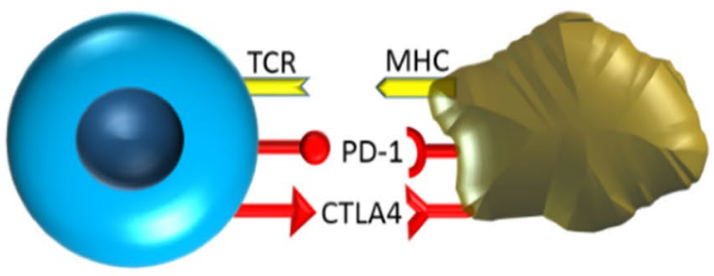

(a)

Inhibitory signals by PD-1 and CTLA4 pathway

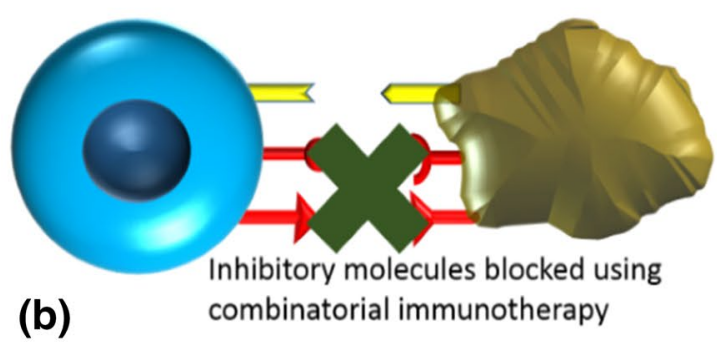

(b)

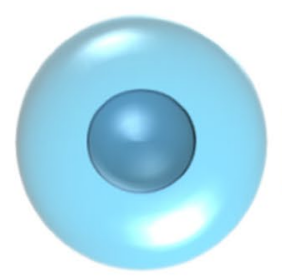

T cell inactivation
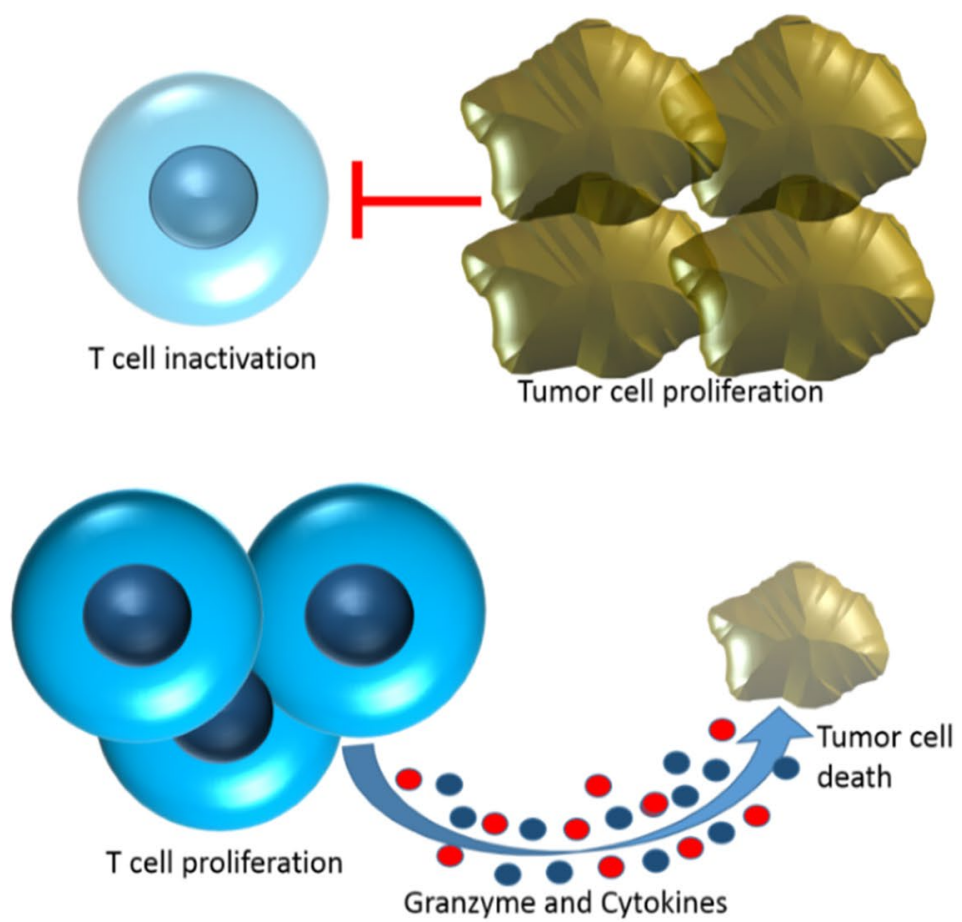

Fig. 1 a Untreated tumor microenvironment with tumor cell proliferation and $\mathrm{T}$ cell inactivation. b Tumor patients treated with combinatorial immunotherapy involving ipilimumab and nivolumab 
ex vivo condition and transferring the CTLs to the patients [79]. The patient undergoes lymphodepletion chemotherapy prior to the introduction of adoptive cells into the patient [73]. The first clinical trial of adoptive cell transfer was MART-1 melanoma antigen targeted transfer of T cells with high affinity TCR. But the problem in this case was, this antigen was not tumor specific and showed severe off target effects like vitiligo and destruction of melanocytes in the eye [77]. CEA was first discovered in 1965, by Gold et al. who demonstrated humoral responses to CEA [65] and has long been a target for immunotherapy, including DNA vaccine development and TCR therapy [31, 33, 69]. Melanoma-associated antigen 3 (MAGE-A3) is a CTA that is specifically expressed in more than $30 \%$ of common epithelial malignancies, including melanoma, breast, lung, esophageal, and head and neck cancers. In a TCR study targeting the MAGE-A3 antigen, two patients undergoing treatment experienced cardiogenic shock, resulting in death. Autopsy revealed severe myocardial damage and histopathological analysis revealed T-cell infiltration. Further study described that MAGE-A3 have a similar structure of a cardiac protein, Titin which might be a possible target for future immunotherapy.

Another technique is genetic engineering of TCR which is highly specific to the tumor antigen, is called Chimeric antigen receptor of $\mathrm{T}$ cells (CAR T cells). In this technique $\mathrm{T}$ cells are obtained from the tumor bearing patients and the desired gene is inserted by lentivirus or retrovirus and then transfused into the patient. As these cells are autologous, there is no risk of GVHD (Graft Versus Host Disease) [39]. Chimeric antigen receptors (CARs) contain a single chain variable region from a monoclonal antibody $(\mathrm{mAb})$, linked to a hinge region, a transmembrane domain, and an intracellular tyrosine-based activation motif (4-1BB or CD28), and TCR intracellular signaling domain. According to the components of the chimeric receptor, CAR can be of different generations [28]. The first generation CAR contains a single-chain variable region from a monoclonal antibody paired with an intracellular signaling domain, the $\mathrm{CD} 3 \zeta$ chain from the CD3 TCR or FcR $\gamma$ [32]. Second generation CAR contains an additional co-stimulator domain and third generation CAR can have two co-stimulator domain (e.g. CD28, 4-1BB, OX-40), that increase signal strength, proliferation and cytokine production [34]. Recently fourth generation CARs have been created (also known as TRUCKs) which have more anti tumorigenic capacity [12]. The principal mechanism of adaptive tumor immunity is killing of tumor cells by CD8+ CTLs. In vivo CD8+ T cell, specific for tumor antigens, may require cross presentation of the tumor antigens by DC cells, as because most tumor cells do not express co-stimulator and low level of MHC I expression. CARs can bypass the need for tumour cells to possess functional antigen processing machinery and express antigen through MHC class I or II molecules, because CAR contain antigen receptor with TCR intracellular domain and proper co-stimulatory molecules. There are certainly some side effects of CAR T cell therapy (neurological toxicity, on target/off tumor recognition) but the most dangerous is cytokine release syndrome (cytokine storm) in which massive T-cell activation triggers production of pro-inflammatory cytokines causing fever, flushing, and dyspnea [32]. The first successful CAR therapies were against B cell malignancies (acute lymphoblastic leukemia, chronic lymphocytic leukemia and many different forms of Hodgkin's lymphoma) [53]. CD19 marker was chosen as target CAR T therapy, as because CD19 is a marker that is expressed only by B cells. The first CAR targeting CD19 was very much effective in a patient with B-cell lymphoma, with concomitant marked lymphoma regression [37]. The first two FDA approved CAR-T therapies (targeting CD19) were approved for relapsed/refractory diffuse large B-cell lymphoma (DLBCL) and relapsed/refractory B-cell precursor acute lymphoblastic leukemia (ALL) [34]. Another experiment on neuroblastoma trial in 2008 was Epstein-Barr virus (EBV)-specific engineered T-cells, co-express a CAR targeted at disialoganglioside GD2, an antigen expressed by neuroblastoma cells. Half the patients (4/8) had tumor regression. It was reported that New York Esophageal Squamous Cell Carcinoma 1 (NYESO-1), a member of the CTA family, is a potential cellular target that is expressed in synovial cell carcinoma (in $80 \%$ of cases) and also less frequently melanoma (in 25\% of cases). Adoptive transfer of CAR T cells specific for NY-ESO-1 antigen showed tumor regression in patients [77]. CAR Ts are more effective on blood cancer rather than solid tumor, as mentioned earlier that solid tumor microenvironment is suppressive, associated with MDSC, TAMs, Tregs, so the generation of armoured CAR T cells which can secrete IL12 (proinflammatory cytokine) improve the efficacy of the therapy [43]. Thus the evolution of CAR T therapy is an ongoing research aspect which can give a better prognosis with better specificity.

\section{APC based immunotherapy}

There are many different strategies applied by tumor cells to escape immune-surveillance. One of them is loss of antigen presentation by antigen presenting cells (APC). So APCs can be used as a major component of active cancer immunotherapy. Especially DC cells which can induce T cells in vivo against tumor antigen can serve as a potent immune therapeutic agent [94].

Many clinical trials (ex. sipuleucel-T) have encouraged therapeutic use of ex vivo generated DC as cancer immunotherapy in metastatic prostate cancer, stage IV melanoma, multiple myeloma etc. From these various studies it has been observed that DC based vaccines are safe and can induce $\mathrm{CD} 4+$ and $\mathrm{CD} 8+\mathrm{T}$ cells that are tumor antigen specific. 
Although therapeutic use of autologous natural APCs is effective but the cost and time to execute this therapy is huge. Therefore, development of artificial APCs (aAPCs) has given an alternative promising approach. The mixed results observed in clinical trials may be due to the less availability of information on the optimal antigen-loaded DC combined with deleterious effects of immunosuppressive factors in the tumor microenvironment [80]. In addition, isolation and ex vivo stimulation of autologous DCs proved time-consuming and expensive, and the quality of ex vivogenerated DCs can be variable [81]. The use of patientderived autologous DCs therefore limits standardization of DC-based treatment procedures. To overcome the disadvantages and difficulties in use of autologous APCs, artificial APCs (aAPCs) have been developed as an alternative for both ex vivo and in vivo induction of tumor-specific CTLs.

Xenogeneic or allogeneic cells, engineered by using retroviral or lentiviral transduction introduce molecules that provide the necessary TCR, costimulatory, and adhesion events required for immune synapse formation. Utilizing this technique, certain cells such as Drosophila cells, murine fibroblasts, and human erythro-leukemia cells, have been used as aAPCs [87]. These cells are easier to handle and are better defined than DCs, allowing far more control over the signals delivered. In addition, cellular aAPCs are stable cell lines that can be stored for extended times and can thus be obtained from a readily accessible source [93].

Different types of synthetic artificial antigen-presenting cells (aAPCs) have been created, for example: (A) Rigid spherical particles: polystyrene latex microbeads, magnetic nano- and microparticles, nanosized quantum dots and poly(lactic-co-glycolic acid) (PLGA) microspheres: (B) Nonspherical particles: carbon nanotube bundles, ellipsoid PLGA microparticles and nanoworms: and (C) Fluidic lipid bilayer-containing systems: 2D-supported lipid bilayers (2D-SLBs), liposomes, RAFTsomes/microdomain liposomes and SLB particles [92, 93].

\section{Future aspect of aAPC development for more potent immunotherapy}

Proper signal presentation by aAPCs and T cell activation are most important aspects of potent immunotherapy. Ex vivo activation of $\mathrm{T}$ cells for subsequent reinfusion into patients has proven most effective with large microsized particles. Application of aAPCs for ex vivo T cell activation, can be improved by using current knowledge about the optimal choice of surface molecules, cytokine release, particle shape, ligand mobility, and ligand orientation. aAPCs that closely mimic features of natural DCs has improved the clinical efficiency of ex vivo-generated T cells.

In vivo, active aAPC immunotherapy does not depend on autologous $\mathrm{T}$ cells, so labor and costs are significantly lower.
The development of nanosized aAPCs with improved flexibility, optimal shape, and efficient signal presentation for direct in vivo aAPC immunotherapy is desirable and holds marked promise.

\section{APC based vaccines}

APC based vaccine was first described by Guo and colleagues, who showed that rats vaccinated with fusions of hepatoma cells and activated B-cells were resistant to subsequent tumor challenge, and that animals with established tumors rejected them, in a manner that was dependent on both CD4+ and CD8+ T-cells. Fusions of antigen presenting cells and tumor cells have been investigated in animal models and phase I/II clinical trials as an effective candidate in the race for cancer vaccines [29].

By fusing tumor cells with professional APCs in vitro, researchers have generated fusion hybrid cells that express tumor-associated antigens (TAAs) and have the capacity to process and present these to the immune system in a manner that induces effective tumor-specific immunity. Trefzer et al. performed an experiment with a fusion cell vaccine of autologous tumor cell and allogenic DC with stage v melanoma patients while another group showed breast or renal cancer regression using autologous DC/tumor cell fusions [93].

EBV B-lymphoblastoid cells (B-LCL) has been used as an alternative to DC as APCs in generating tumor hybrid cell lines [108]. Unlike in vitro generated DCs, EBV B-LCL are immortalised for growth in cell culture. Stimulation of (allogeneic) peripheral blood T-cells from both healthy donors and tumor-bearing patients in vitro using LCL/tumor hybrid cell lines induced tumor antigen-specific CTLs that killed tumor cells, demonstrating the potential of these hybrid cell lines to induce tumor-specific immune responses in humans, in vitro at least [108].

In summary, the use of APC/tumor cell fusion vaccines on phase I/II animal models have been done over two decades. But the efficacy of APC/tumor cell fusion vaccines in human patients has been much less promising than that have been found in animal models. So to validate the therapy in human further investigation regarding optimization of the treatment procedure must be done (Fig. 2).

\section{New era of immunotherapy: T regulatory cells}

Immune systems play a pivotal role in recognizing cancer and induce effective immune responses for their clearance. Avoidance of immune system is one of the major hallmarks in cancer progression that successively transforms immune surveillance (tumor eradication) to immune tolerance (tumor progression) [21]. Modulation of immune cells to harness 


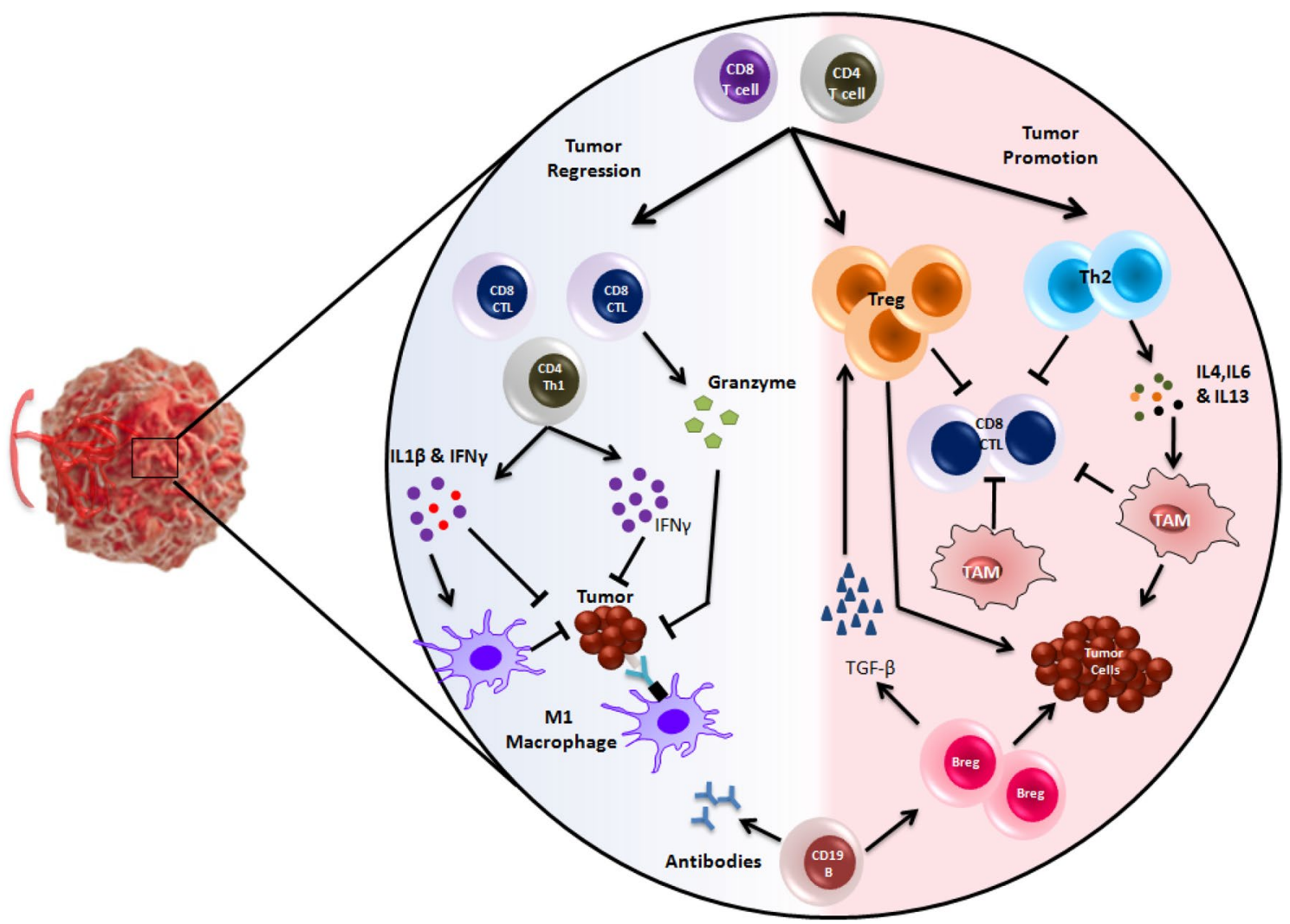

Fig. 2 A detailed view of the tumor microenvironment. The left hand (blue color) side represents the normal condition where the immune cells are behaving in anti-tumorogenic manner, thereby killing the tumor cells and preventing their growth. The right hand (red color) side represents the tolerogenic condition of the immune cells in tumor

the power of effective immune responses has been long-term goals for promising strategies of cancer immune therapy. Cancer immunotherapy involving blockade of immune checkpoint molecules, such as CTLA-4 and PD-1, has shown remarkable clinical success across several types of malignancies. However, a large fraction of patients experience disease progression after treatment; thus, exploring resistant mechanisms for immune checkpoint inhibitors and improving their treatment outcome with additional modalities are of great importance [10, 67]. Long-term follow-up in a pooled meta-analysis exhibited long-term survival in approximately $20 \%$ of patients treated with immune checkpoint inhibitors. CD4+ T-regulatory (Treg) cells characterized by expression of the master regulatory transcription factor FOXP3 are a highly immune-suppressive subset of CD4+ T cells that maintain immune homeostasis. Several preclinical and clinical studies suggest that Treg cells hamper immune surveillance against cancer in healthy individuals, prevent the development of effective antitumor immunity in tumor-bearing patients, and promote tumor progression [8]. Therefore, targeting Treg cells should be crucial to improving the treatment outcomes of cancer immunotherapy. Understanding microenvironment where they help the tumor cells to propagate and grow in number. TAM Tumor associated macrophage, Breg B regulatory cell, CTL Cytotoxic lymphocytes, Treg T regulatory cells (color figure online)

the characteristics and roles of Treg cells in cancer settings could make disease-specific Treg-targeted therapy more efficacious and reduce the incidence of immune-related adverse effects mediated by Treg cell inhibition.

\section{Treg plasticity}

Understanding the plasticity of Treg cells in tumor microenvironment is also important to characterize them and to decipher their role in tumor progression. The stability of Treg lineage is complicated and FOXP3+ Treg cells can convert themselves into $\mathrm{T}$ helper subsets and vice versa under suitable condition. The tumor microenvironment contain angiogenic factors and/or suppressive immune cells and may be hypoxic and all this factors help in the growth of tumor cells [61]. It has been documented numerous times in multiple cancers that the tumor microenvironment is highly enriched in Treg cells compared to non-tumor surrounding tissue [26, 99]. Tumor-infiltrating Tregs have an eTreg phenotype (FOXP3hiCD45RA-), and some markers which are associated with enhanced suppression (CTLA-4, ICOS) and activation (CD25, CD69); are up-regulated in those tumor 
infiltrating Treg cells and these cells become more suppressive than those from non-tumor tissue from the same patient [11]. These results indicate that factors within the tumor microenvironment lead to recruitment of Tregs to tumors and enhance the suppressive activity of Tregs and these factors results in the plasticity of Treg cells.

\section{Metabolism}

To mount the immune responses, the immune cells need to rapidly grow, divide, and produce cytokine and this requires different bio-energetic processes [25]. Cell metabolism controls the pathways that are involved in regulating immune cell functions and differentiation and thus modulation of Treg cell metabolism is currently of high therapeutic interest. As tumor grows, as a result of increased aerobic glycolysis by tumor cells, glucose is depleted and lactate accumulation occurs in extracellular tissue [70]. These conditions i.e., decreased nutrient availability suppress the activity of T-effector cells, but Treg cells survive this inhospitable environment and supports the growth of tumor. Modulation of Treg cell metabolism is currently of high therapeutic interest [61] because these cells are necessary for maintaining immune tolerance to self tissues, but they turn out to be harmful to the body in case of tumor development. The primary fuel source for generation of ATP is glucose for the $\mathrm{T}$ cells [98]. The resting $\mathrm{T}$ cells (naïve, memory and anergic $\mathrm{T}$ cells) have low metabolic requirements and therefore, maintain a metabolic balance that supports basal energy production over biosynthesis [24]. Approximately 96\% of the required ATP for naïveT cells are generated via mitochondrial oxidative phosphorylation (OXPHOS) pathway [38]. Whereas, upon stimulation, the activated T cells divide and differentiate to generate various $T$ cell subsets and each subset requires distinctive metabolic pathways for their energetics [41]. During proliferation, these cells require high amount of ATP and the other precursors of protein, lipids, nucleic acid etc. [41]. To meet the requirement they shift from catabolic mitochondrial OXPHOS pathway to glycolytic and other anabolic pathways. Though aerobic glycolysis is less efficient than OXPHOS in generating ATP per molecule of glucose, still activated $\mathrm{T}$ cells and highly proliferating cancer cells utilize this pathway for their energetics which is known as "Warburg effect" $[41,50]$. This unusual metabolic features, although is less efficient in energy production, provides the raw materials for the synthesis of protein, lipid, nucleic acid etc. [42]. Interestingly, differentiated $T$ cells utilize distinct energetic and biosynthetic pathways to support the specific functional needs [40, 96]. Recent studies indicated that different subsets of $\mathrm{T}$ cell do not require uniform metabolic programming. CD4+ Teff cells like, Th1, Th2, Th17, depends on aerobic glycolysis whereas the induced-Treg (iTreg) cells have been shown to be less dependent on aerobic glycolytic pathways [5, 105]. The tumor microenvironment become unfavorable for T-effector cells whereas iTreg cells can survive in that condition and further suppress the T-effector cells and thus help in the progression of the tumor. Understanding the metabolomics of Treg cells in tumor microenvironment will give the clue to manipulate these immunosuppressive pro-tumor Treg cells to check tumor progression.

\section{Molecular pathways to target Treg}

In a normal individual, the Treg and the effector $\mathrm{T}$ cells remain in a homeostasis where the effect of Treg is prominent enough to prevent autoimmunity in the individual. However, during any event of infection, the Treg population is destabilized to accommodate for the enhanced functioning of the effector $\mathrm{T}$ cells in order to combat the infection [67]. This process of destabilizing of the Treg is tightly controlled and mainly involves specific local pro inflammatory signals like IL6 and IFN $\gamma$ [58]. Other factors contributing to the destabilization process of Tregs are PD1, Helios and Neuropilin1 [58]. On the other hand, there are certain pathways which prevent this destabilizing of Tregs and help them maintain their suppressive actions. The recently discovered PTEN pathway and the immune suppressive enzyme IDO plays a vital role preventing destabilization of Treg cells.

Hence these pathways are often targeted as modes of immunotherapy to decrease the tolerogenic properties of Treg in tumor microenvironment. It has been seen in clinical trials that patients treated with anti IL6 antibody tocilizumab develop acute inflammation and decreased Treg suppressive activity [35]. Loss in IL6 receptor chain causes abrogation of anti-tumor efficacy in tumor bearing mice [85]. IFN $\gamma$, another pro inflammatory cytokine with the help of Neuropilin-1 (Nrp1) helps in destabilizing the tumor infiltrating Tregs and trigger the anti-tumor response in the body [64]. Another pathway of destabilizing Treg in tumor micro environment is the PI3K-Akt-mTOR pathway which results in loss of Foxp3 functioning thereby sharply decreasing the Treg suppressive activity [36]. This pathway is now embattled through drugs like the PI3 K inhibitors LY294002 and Wortmannin, both targeting the catalytic site of p110 and have been extensively used as research tools on tumor bearing murine models (cancer cell), though any clinical trials using this pathway has not yet been planned [58]. Helios, one of the major transcription factors of Treg cell when down regulated cause disruption of Foxp3 and thereby help in destabilizing the Treg suppressive action in tumor microenvironment [58]. New researches are targeting helios as a switch to alter the suppressive functions of Treg.

The involvement of MicroRNAs in the Treg functions and maintenance has also been targeted by scientists in recent times. The miR-155 was found to have a major effect 
on the suppressive character of Treg cells [107]. Certain other microRNAs like miR-226 and miR-146 has also been reported to be up regulated in Treg cells [14] though their exact role is not yet identified. However, other studies have reported that, deactivating the Drosha and Dicer complex in Treg cells severely hampers the normal functioning of the Treg cells $[13,14]$. Newer avenues of immunotherapy are emerging which might target microRNAs specific to Treg and involved in its suppressive functions to prevent the Treg based immune tolerance in tumor micro environment [30].

\section{Treg recruitment in tumor microenvironment}

Migration and retention of Tregs in different body compartments is necessary to maintain immune homeostasis. CD4+ CD25+ FOXP3+ regulatory $\mathrm{T}$ cells differentiate in thymus and from the thymus they move to the peripheral circulation in response to different chemokines. Tregs compartmentalization and trafficking is dependent upon distinct chemokine and integrin expression. Several authors have shown the recruitment of Tregs in different cancers including ovarian cancer [16], breast cancer [27]. Tregs express CCR4 that helps in the recruitment of these cells in tumor. CCR4+ Tregs migrate towards tumor micro-environmental CCL22 released by tumor cells and tumor associated macrophages. These tumor Treg cells are functionally suppressive and able to block tumor-specific immunity, foster tumor growth, and predict poor patient survival [16]. Other than CCR4, CCR8 also acts as an important receptor for recruitment of Tregs in tumor [71]. Recent studies have demonstrated that CCR8 is uniquely up-regulated in human tumor-resident Tregs of breast, colon, and lung cancer patients when compared to normal tissue-resident Tregs. Therefore, CCR8+ tumor-resident Tregs are rational targets for cancer immunotherapy [95]. Depletion of CCR4-expressing FoxP3+ CD4 Tregs by KW-0761 infusion was investigated in solid cancer patients with promising results [44]. It has also been reported that anti-CCR4 mAb treatment is instrumental for evoking and augmenting antitumor immunity in cancer patients by selectively depleting eTreg cells [89]. Mogamulizumab, an antiCC chemokine receptor 4 (CCR4) monoclonal antibody, has also shown very promising results for CCR4-positive T-cell lymphomas, especially for ATL, proving that the current pathway of immunotherapy is guided by targeting the key modulators of Treg cells [49] (Fig. 3).

\section{Novel player in the arena: B cell subtypes}

$\mathrm{T}$ cell has been the major area in the context of the tumor immunology research for decades. However, this concept is changing with the emergence of a new theory. The potential contribution of $\mathrm{B}$ cells along with $\mathrm{T}$ cells and their correlation of fighting against tumor antigens has opened up a new dimension in the field of tumor immunology. B cells are a heterogeneous population, contributing to pro as well as the anti-tumorigenic effect on tumor development and their behavior [52]. B cells can contribute to immune responses through various mechanisms. Being a potent antigen-presenting cell, they play a deciding role in the activation of Th1 cells thereby helping in the secretion of IFN gamma from the cell type. One of the major weapons that the $\mathrm{B}$ cells possess is their antibody-producing capacity. These antibodies bind to antigen, thereby activating the complement system cascade as well as the antibody-dependent cellular cytotoxicity (ADCC) pathway by associating with the natural killer (NK) cells. B cells are also known for the pro-inflammatory cytokines secreting capability, such as granulocyte-macrophage colony-stimulating factor (GM-CSF), tumour necrosis factor (TNF), that leads to the activation of the myeloid lineage cells and subsequently drive the polarization balance between T helper 1 (TH1) cell and TH17 cell responses [82, 101, 103]. Tumor-infiltrating B cells (TIL-Bs) are one of the most critical aspects of the B cell response towards cancer. TIL-Bs make up to $40 \%$ of the tumor infiltrating lymphocytes (TILs) in breast tumor as well as $25 \%$ in other tumor tissues [15, 51]. High grade serous ovarian cancers also contain $40 \%$ of CD20+ B cells [60]. Most of the B cells are found in the tumor-draining lymph nodes of mice, suggesting their role in the context of tumor modulation [47]. It was also observed that TIL containing CD20+ B cells as wells as CD8+ killer $\mathrm{T}$ cells have more survival rate than the TILs containing only CD20+ B cell or CD8+ T cells and the association of different $\mathrm{T}$ cell markers such as granzyme, TRAIL, etc. with the B cell made the cell-mediated immune system even more stronger to fight against tumor antigens $[1,57$, 75]. One of the vital phenomena for B cell is their ability to produce antibody upon encountering an antigen, and the TIL B has no exception in this case [106]. Antibodies derived from those cells bind on the tumor-specific antigen of mice and destroy them through complement-dependent lysis pathway or by Antibody-dependent cell-mediated cytotoxicity (ADCC) [47]. Blockade of co-stimulatory molecule like CTLA4 or PD1-PDL1 on T cell enhances the proliferation of memory B cells and their antibody production rate $[19,23,72]$. It was also reported that regression of tumor occurs when mice are treated with tumor antigen binding natural $\mathrm{IgG}$ or with the activation of dendritic cells (DCs) [102]. The effectiveness of tumor regression become way stronger when tumor-bearing mice treated with the both [38]. Beside the antitumor response of B cell, they also produce interleukin-10 (IL10), which help in tumor progression by suppressing the conventional $\mathrm{T}$ cells [9]. This paradox of B cell biology introduced us 
Fig. 3 The multi-edged facet of T-regulatory cell in promoting the suppressive functions in (TME) the tumor micro environment

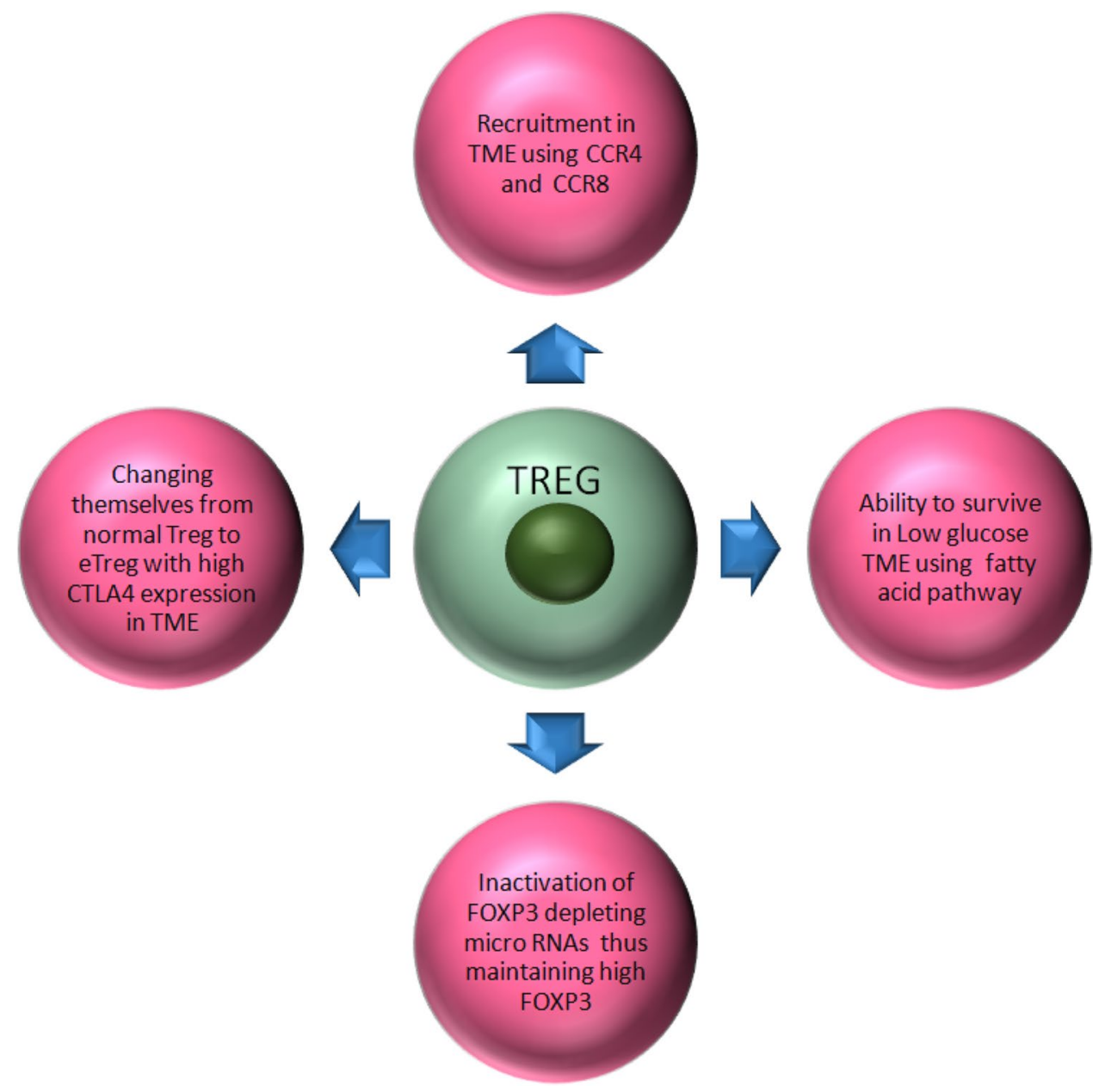

to a new subset of immunosuppressive B cells known as Regulatory B (Breg) cells [6]. Regulatory B (Breg) cells are a group of immunosuppressive cells that support immunological tolerance through the secretion of interleukin-10 (IL-10) [54]. Although IL10 is the major immunosuppressive cytokine of B regulatory cells, other immunosuppressive agents such as PDL1, FasL, IL-35, Tgf-B have also been reported for their immunoregulatory function. Breg activation requires the toll-like receptors rather than the antigen receptor [18, 79]. This group of suppressive cells also facilitates the recruitment of Treg (another group of the immunosuppressive cell). Tumorassociated T cell-secreted IL2, IL1B, IL6 are also known to induce Breg. IL-35 production by the Breg cells is the recent breakthrough in this field. IL-35 can inhibit experimental uveitis on adoptive transfer [48]. The Breg also found in the peripheral blood and within the tumor microenvironment which was demonstrated to suppress T-cell and natural killer cell responses. Thus it interferes with both, i.e., acquired and innate immunity [84]. B celldirected therapies include the use of monoclonal antibodies (MEDI-551 rituximab, ofatumumab, obinutuzumab,
Epratuzumab) against B cell surface markers, such as CD19, CD20, CD22. However, the trialdid not see a high success rate because of the reducing number of short-lived plasmoblasts and memory B cell subsets and low antibody production rate $[97,108]$.

It is already established that Breg cells create substantial hindrance in cancer therapy. During tumor, it was reported that regulatory $\mathrm{B}$ (Breg) cells are increasing in number and the heavy amount of IL10, transforming growth factor- $\beta$ (TGF $\beta$ ), and indoleamine 2,3-dioxygenase (IDO) secretion, as well as the activation of programmed cell death ligand 1 molecule (PDL1), leads to a complete immune disruption [108]. Few reports suggest that CD20low4-1BBLlow immune-suppressive Breg is responsible for the failure of rituximab whereas IgA + CD138+ PD-L1+ IL-10+ Breg subset impedes the chemotherapeutic drug oxaliplatin-mediated expansion of anti-tumor CTL [7]. Depletion of CD20+ $B$ cell with anti-CD20 Ab causes an increased percentage of the Breg in 4T1 tumor-bearing mice [3]. This tumor-specific Breg also increases metastasis progression by enhancing the $\mathrm{T}$ cell-mediated suppression. So by reducing the numbers of regulatory $\mathrm{B}$ (Breg) cells in the tumor, a promotion in 
pro-inflammatory cytokine circuits and a higher antibody production level is expected by activating the $\mathrm{T}$ helper 1 (TH1) cells, TH17 cells, and the conventional B cells. Considering the lack of information in this particular topic, further work in Breg depletion and the signals that drive Breg differentiation might open new avenues in searching for drug targets to develop successful immunotherapy against cancer.

\section{Immunotherapy: challenges and future}

Though immunotherapy has proved itself to be an effective tool against tumour but there is still a lot of limitations on its way. It has been seen that immunotherapy and mostly the traditional mono-immunotherapy has failed in many cases based upon the type of cancer it is used against and the population in which it is used $[2,84,100]$. Some of these therapies has generated an array of autoimmune like inflammatory phenomenon while others have shown inclination to prohibitive toxicity and erroneous conclusion about the therapeutic potential and the risk-benefit condition of the combinatorial therapy $[59,63]$. These results, along with other failures have led to very few clinical trials in the field of immunotherapy. Also in certain cases, Phase I trials do not yield good results and the drugs are not further tried [4]. Nowadays, innovative trial designs are used to prevent further failures, as novel combinations present the possibility of enhanced efficacy compared with mono-therapies [22].

The failure of older methods of immunotherapy has caused the inclusion of demonstrations that no new toxicities develop due to any combination with the mono-therapies. The combination of ipilimumab with vemurafenib and ipilimumab with dacarbazine have also been found to develop hepatic enzyme elevation in the majority of patients and also showed much higher toxicity than was expected [74, 78]. Certain algorithms has been developed for the investigation of the possible outcomes of these combinations and use of these algorithms has proved to be quite fruitful in preventing any side effect related deaths in combinatorial immunotherapies [45].

Some mono-therapies may show some immediate effects on the patients but when the pattern by modified World Health Organization (mWHO) or Response Evaluation Criteria in Solid Tumors (RECIST) assessment is used for the evaluation of the efficacy of the treatment, most of the immunotherapies fail in producing any considerable results in the long term [7]. However, a combinatorial therapy involving anti PD-1 drugs like ipilimumab and anti CTLA-4 drugs nivolumab has been used extensively in melanoma and in patients with NSCLC in various combination for enhanced clinical effect and improved safety with satisfactory results [76]. Careful analysis using the RECIST technique has revealed that the $20 \%$ of population of patients treated by the combinatorial therapy who failed to show any visible improvement, eventually had anti-tumor response and long term stabilization thereby having long term survival rate [76]. Similar pattern of late response and loner survival has also been reported in patients infected with oncolytic virus, T-VEC and melanoma patients [55]. These findings suggest that newer models of immunotherapy with combination of new, efficient drugs combined with algorithm testing for possible side effects before clinical trials will not only provide a complete haul of the present immunotherapy thereby providing better chances of survival but will also increase the stabilization of the patients in long term [83]. WHO has also introduced a new criteria called the "durable response rate" (DRR) while testing the efficacy of new combinatorial immunotherapies to ensure that these immunotherapies do not produce any side effects even in the long run [7]. Recently Food and Drug Administration (FDA) approved "next generation drugs" ofatumumab and obinutuzumab which are anti-CD20 Abs for the betterment of human civilization to fight against the deadly tumor [104].

The number of cancer patients who have been benefitted from the immunotherapy has increased considerably over the past decade. With researches on immunotherapy being on the rise all over the globe, further improvements are soon going to come in the field of immunotherapy thereby reducing the adverse side effects and increasing the efficacy of the immunotherapy as a whole. Currently, we are at the doorstep of entering a thrilling era of combinatorial and cuttingedge immunotherapy that bids the prospect to construct on the proofs that has been derived from clinical experience and form a new world of fool proof immunotherapy where the side effects will be minimal, if not nil and the effect on tumor will be maximal with high survival rate of patients. However, a number of considerations need to be kept in mind in this progress towards the future of immunotherapy. Firstly, a severe calculation of the optimal dose, sequence should be done in both the algorithmic models and in the clinical settings. Secondly, it should be addressed that some compounds might have much lesser side effects in mono therapy but have a lot more side effects when administered in combination with other drugs. Thirdly, multiple indications should be kept in mind and a flexible approach should be taken while dose selection and the decision making as well as application of the drugs on patients. Fourthly, supportive care of patients as well as education of the family members of patient regarding the identification and importance of immune related adverse effects (irAE) should be given careful attention. Lastly, clinical trials should only be started after efficient testing of the safety and clinical activity of the drugs under algorithmic simulations.

There is incredible potential in immunotherapy particularly with the advent of combinatorial therapies and newer players in the arena like Treg and Breg which might prove 
to be one of the key factors in controlling the immune responses in the tumor microenvironment. The increasing failures of $\mathrm{T}$ cell based therapies like adoptive transfers and CAR-T based therapies, it makes it necessary to target newer molecules and approach the tumor immunology in a holistic approach. Newer researches and uncovering of novel molecules performing major functions is providing that much needed stones to fill up the gap of theoretical explanations and failed clinical trials. Targeting these molecules will provide a much better focus to improve the immunotherapy field and fulfill the needs of the patients all over the globe in very near future.

Acknowledgements This work was supported by research grants from Department of Science and Technology, Govt. of India, University Grants Commission and Council of Scientific and Industrial Research, India.

\section{Compliance with ethical standards}

Conflict of interest The authors declare no conflict of interest.

\section{References}

1. Al-Shibli KI, Donnem T, Al-Saad S, Persson M, Bremnes RM, Busund LT. Prognostic effect of epithelial and stromal lymphocyte infiltration in non-small cell lung cancer. Clin Cancer Res. 2008;14:5220-7.

2. Antonia SJ, Gettinger SN, Chow LQM, Juergens RA, Borghaei H, Shen Y. Nivolumab (anti-PD-1; BMS-936558, ONO-4538) and ipilimumab in first-lineNSCLC: interim phase I results. J Clin Oncol. 2014:32:74-86.

3. Au KM, Tripathy A, Lin CP. Bespoke pre targeted nanoradioimmunotherapy for the treatment of non-hodgkin lymphoma. ACS Nano. 2018;83:48-59.

4. Bates SE, Berry DA, Balasubramaniam S, Bailey S, LoRusso PM, Rubin EH. Advancing clinical trials to streamline drug development. Clin Cancer Res. 2015;21(20):4527-35.

5. Beier U, et al. Essential role of mitochondrial energy metabolism in Foxp3+ T-regulatory cell function and allograft survival. FASEB J. 2015;29:2315-26.

6. Berthelot J-M. Regulatory B cells play a key role in immune system balance. Jt Bone Spine Rev Rhum. 2013;80(1):18-22.

7. Bodogai M, Lee Chang C, Wejksza K. Anti-CD20 antibody promotes cancer escape via enrichment of tumor-evoked regulatory B cells expressing low levels of CD20 and CD137L. Cancer Res. 2013;73(7):2127-38.

8. Bose S, Panda AK, Mukherjee S, Sa G. Curcumin and tumor immune-editing: resurrecting the immune system. Cell Div. 2015;10:6-30.

9. Carmi Y. Allogeneic $\operatorname{IgG}$ combined with dendritic cell stimuli induce antitumour T-cell immunity. Nature. 2015;521:99-104.

10. Chakraborty S, Panda AK, Bose S, Roy D, Kajal K, Guha D, Sa G. Transcriptional regulation of FOXP3 requires integrated activation of both promoter and CNS regions in tumor-induced CD8 ${ }^{+}$Treg cells. Sci Rep. 2017;7:1628-9.

11. Chaudhary B, Elkord E. Regulatory T cells in the tumor microenvironment and cancer progression: role and therapeutic targeting. Vaccines. 2016;4:28-31.
12. Chmielewski M, Abken H. TRUCKs: the fourth generation of CARs. Expert Opin Biol. 2015;15(8):1145-54.

13. Chong MM, Rasmussen JP, Rudensky AY, Littman DR. The RNAseIII enzyme Drosha is critical in T cells for preventing lethal inflammatory disease. J Exp Med. 2008;205(9):2005-17.

14. Cobb BS, Hertweck A, Smith J, O'Connor E, Graf D, Cook T, Smale ST, Sakaguchi S, Livesey FJ, Fisher AG, Merkenschlager M. A role for Dicer in immune regulation. J Exp Med. 2006;203:2519-27.

15. Coronella-Wood JA, Hersh EM. Naturally occurring B-cell responses to breast cancer. Cancer Immunol Immunother. 2003;52:715-38.

16. Curiel TJ, Coukos G, Zou L. Specific recruitment of regulatory $\mathrm{T}$ cells in ovarian carcinoma fosters immune privilege and predicts reduced survival. Nat Med. 2004;10:942-9.

17. Curiel TJ. Tregs and rethinking cancer immunotherapy. J Clin Investig. 2007;117:1167-74.

18. Dambuza IM. IL-12p35 induces expansion of IL-10 and IL35 -expressing regulatory $\mathrm{B}$ cells and ameliorates autoimmune disease. Nat Commun. 2017;8(1):719-29.

19. Dong H, et al. Tumor-associated B7-H1 promotes T-cell apoptosis: a potential mechanism of immune evasion. Nat Med. 2002;8:1039.

20. Dong H, Strome SE, Salomao DR, Tamura H, Hirano F, Flies DB, et al. Tumor-associated B7-H1 promotes T-cell apoptosis: a potential mechanism of immune evasion. Nat Med. 2002;8:793-800.

21. Dunn GP, Old LJ, Schreiber RD. The three Es of cancer immunoediting. Annu Rev Immunol. 2004;22:329-60.

22. Emens LA, Butterfield LH, Hodi FS Jr, Marincola FM, Kaufman HL. Cancerimmunotherapy trials: leading a paradigm shift in drug development. J Immunother Cancer. 2016;4:42-51.

23. Fanoni D. New monoclonal antibodies against B-cell antigens: possible new strategies for diagnosis of primary cutaneous B-cell lymphomas. Immunol Lett. 2011;134:157-60.

24. Frezza C, Mauro C. Editorial: the metabolic challenges of immune cells in health and disease. Front Immunol. 2015;6:293.

25. Gerriets VA, Danzaki K, Kishton RJ, Eisner W, Nichols AG, Saucillo DC, Shinohara ML, MacIver NJ. Leptin directly promotes T-cell glycolytic metabolism to drive effector T-cell differentiation in a mouse model of autoimmunity. Eur J Immunol. 2016;46(8):1970-83.

26. Girardin A, McCall J, Black MA, Edwards F, Phillips V, Taylor ES, et al. Inflammatory and regulatory $\mathrm{T}$ cells contribute to a unique immune microenvironment in tumor tissue of colorectal cancer patients. Int J Cancer. 2013;132:1842-50.

27. Gobert M, Treilleux I, Bendriss-Vermare N, Bachelot T, Goddard-Leon S, Arfi V, Biota C, Doffin AC, Durand I, Olive D, Perez S, Pasqual N, Faure C, Ray-Coquard I, Puisieux A, Caux C, Blay JY, Ménétrier-Caux C. Regulatory T cells recruited through CCL22/CCR1 are selectively activated in lymphoid infiltrates surrounding primary breast tumors and lead to an adverse clinical outcome. Cancer Res. 2009;69(5):2000-9.

28. Gross G, Gorochov G, Waks T, Eshhar Z. Generation of effector $\mathrm{T}$ cells expressing chimeric $\mathrm{T}$ cell receptor with antibody typespecificity. Transpl Proc. 1989;21:127-30.

29. Guo Y, Wu M, Chen H, Wang X, Liu G, Li G, et al. Effective tumor vaccine generated by fusion of hepatoma cells with activated B cells. Science. 1994;263:518-20.

30. Ha T-Y. The role of MicroRNAs in regulatory $\mathrm{T}$ cells and in the immune response. Immune Netw. 2011;11(1):11-41.

31. Hance KW, Zeytin HE, Greiner JW. Mouse models expressing human carcinoembryonic antigen (CEA) as a transgene: evaluation of CEA based cancer vaccines. Mutat Res, Fundam Mol Mech Mutagen. 2005;576:132-54. 
32. Hartmann J, Schüßler-Lenz M, Bondanza A, Buchholz CJ. Clinical development of CAR T cells-challenges and opportunities in translating innovative treatment concepts. EMBO Mol Med. 2017;9(9):1183-97.

33. Haupt K, Roggendorf M, Mann K. The potential of DNA vaccination against tumor-associated antigens for antitumor therapy. Exp Biol Med. 2002;227:227-37.

34. Houot R, Schultz LM, Marabelle A, Kohrt H. T-cell-based Immunotherapy: adoptive cell transfer and checkpoint inhibition. Cancer Immunol Res. 2015;10:1115-22.

35. Hunter CA, Jones SA. IL-6 as a keystone cytokine in health and disease. Nat Immunol. 2015;16:448-57.

36. Huynh A, DuPage M, Priyadharshini B, Sage PT, Quiros J, Borges CM. Control of PI(3) kinase in Treg cells maintains homeostasis and lineage stability. Nat Immunol. 2015;16:188-96.

37. Kochenderfer JN, Wilson WH, Janik JE, Dudley ME, StetlerStevenson M, Feldman SA, Maric I, Raffeld M, Nathan D-AN, Lanier BJ, Morgan RA, Rosenberg SA. Eradication of B-lineage cells and regression of lymphoma in a patient treated with autologous $\mathrm{T}$ cells genetically engineered to recognize CD19. Blood. 2010;116:4099-102.

38. Korman AJ, et al. Checkpoint blockade in cancer immunotherapy. Adv Immunol. 2006;90:297-339.

39. Korngold R, Sprent J. Graft-versus-host disease in experimental allogeneic bone marrow transplantation. Proc Soc Exp Biol Med. 1991;197:12-8.

40. Kouidhi S, Noman MZ, Kieda C, Elgaaied AB, Chouaib S. Intrinsic and tumor microenvironment-induced metabolism adaptations of $\mathrm{T}$ cells and impact on their differentiation and function. Front Immunol. 2016;7:114.

41. Kouidhi S, Zaeem MN, Kieda C, Elgaaied EB, Chouaib S. Intrinsic and tumor microenvironment-induced metabolism adaptations of $\mathrm{T}$ cells and impact on their differentiation and function. Front Immunol. 2016;7:114-21.

42. Krummel MF, Allison JP. CD28 and CTLA-4 have opposing effects on the response of T cells to stimulation. J Exp Med. 1995;182:459-65.

43. Kueberuwa G, Kalaitsidou M, Cheadle E, Hawkins RE, Gilham DE. CD19 CAR T cells expressing IL-12 eradicate lymphoma in fully lymphoreplete mice through induction of host immunity. Mol Ther Oncol. 2018;8:41-51.

44. Kurose K, Ohue Y, Wada H, Iida S, Ishida T, Kojima T, Doi T, Suzuki S, Isobe M, Funakoshi T, Kakimi K, Nishikawa H, Udono H, Oka M, Ueda R, Nakayama E. Phase I: a Study of FoxP3+ CD4 Treg depletion by infusion of a humanized antiCCR4 antibody, KW-0761, in cancer patients. Clin Cancer Res. 2015;21(19):4327-36.

45. Larkin J, Chiarion-Sileni V, Gonzalez R, Grob JJ, Cowey CL, Lao $\mathrm{CD}$, et al. Combined nivolumab and ipilimumab or monotherapy in untreated melanoma. N Engl J Med. 2015;373(1):23-34.

46. Leach DR, Krummel MF, Allison JP. Enhancement of antitumor immunity by CTLA-4 blockade. Science. 1996;271:1734-6.

47. Li Q, et al. Simultaneous targeting of CD3 on T cells and CD40 on $\mathrm{B}$ or dendritic cells augments the antitumor reactivity of tumor-primed lymph node cells. J Immunol. 2005;175:1424-32.

48. Lin W, et al. Human regulatory B cells combine phenotypic and genetic hallmarks with a distinct differentiation fate. J Immunol Baltim Md. 2014;193(5):2258-66.

49. Makita S, Tobinai K. Mogamulizumab for the treatment of T-cell lymphoma. Expert Opin Biol Ther. 2017;17(9):1145-53.

50. Marelli-Berg FM, Fu H, Mauro C. Molecular mechanisms of metabolic reprogramming in proliferating cells: implications for T-cell-mediated immunity. Immunology. 2012;136(4):363-9.

51. Marsigliante S. Computerised counting of tumour infiltrating lymphocytes in 90 breast cancer specimens. Cancer Lett. 1999;139:33-41.
52. Martin F, Chan AC. B cell immunobiology in disease: evolving concepts from the clinic. Annu Rev Immunol. 2006;24:467-96.

53. Maude SL, Teachey DT, Porter DL, Grupp SA. CD19-targeted chimeric antigen receptor T-cell therapy for acute lymphoblastic leukemia. Blood. 2015;125(26):4017-23.

54. Mauri C, Menon M. The expanding family of regulatory B cells. Int Immunol. 2015;27(10):479-86.

55. Melero I, Hirschhorn-Cymerman D, Morales-Kastresana A, Sanmamed MF, Wolchok JD. Agonist antibodies to TNFR molecules that costimulate T and NKcells. Clin Cancer Res. 2013;19(5):1044-53.

56. Mellman I, Coukos G, Dranoff G. Cancer immunotherapy comes of age. Nature. 2011;480:480-9.

57. Milne K, Köbel M, Kalloger SE, Barnes RO, Gao D, Gilks $\mathrm{CB}$, Watson PH, Nelson BH. Systematic analysis of immune infiltrates in high-grade serous ovarian cancer reveals CD20, FoxP3 and TIA-1 as positive prognostic factors. PLoS ONE. 2009;4:41-62.

58. Munn DH, Sharma MD, Johnson TS. Treg destabilization and reprogramming: implications for cancer immunotherapy. Cancer Res. 2018;78(18):5191-9.

59. Naidoo J, Page DB, Li BT, Connell LC, Schindler K, Lacouture ME. Toxicities of the anti-PD-1 and anti-PD-L1 immune checkpoint antibodies. Ann Oncol. 2015;26(12):2375-91.

60. Nelson BH. CD20+ B cells: the other tumor-infiltrating lymphocytes. J Immunol. 2010;185:4977-82.

61. Norton SE, Ward-Hartstonge KA, Taylor ES, Kemp RA. Immune cell interplay in colorectal cancer prognosis. World J Gastrointest Oncol. 2015;7:221-32.

62. Ott PA, Hodi FS, Kaufman HL, Wigginton JM, Wolchok JD. Combination immunotherapy: a road map. J Immunother Cancer. 2017;5:16.

63. Ott PA, Hodi FS, Kaufman HL, Wigginton JM, Wolchok JD. Combinationimmunotherapy: a road map. J Immunother Cancer. 2017;5:16.

64. Overacre-Delgoffe AE, Chikina M, Dadey RE, Yano H, Brunazzi EA, Shayan G. Interferon-gamma drives treg fragility to promote anti-tumorimmunity. Cell. 2017;169:1130-41.

65. Palmer DC, Chan CC, Gattinoni L, Wrzesinski C, Paulos CM, Hinrichs CS. Effective tumor treatment targeting a melanoma/ melanocyte-associated antigen triggers severe ocular autoimmunity. Proc Natl Acad Sci USA. 2008;105:8061-6.

66. Panda AK, Bose S, Sarkar T, Chakraborty D, Chakraborty S, Sarkar I, Sa G. Cancer-immune therapy: restoration of immune response in cancer by immune cell modulation. Nucleus. 2017;60:93-109.

67. Panda AK, Chakraborty D, Sarkar I, Khan T, Sa G. New insights into therapeutic activity and anticancer properties of curcumin. J Exp Pharmacol. 2017;9:4528-31.

68. Pardoll DM. Immunology beats cancer: a blueprint for successful translation. Nat Immunol. 2012;13:1129-32.

69. Parkhurst MR, Yang JC, Langan RC, Dudley ME, Nathan D-AN, Feldman SA, Davis JL, Morgan RA, Merino MJ, Sherry RM, Hughes MS, Kammula US, Phan GQ, Lim RM, Wank SA, Restifo NP, Robbins PF, Laurencot CM, Rosenberg SA. T cells targeting carcinoembryonic antigen can mediate regression of metastatic colorectal cancer but induce severe transient colitis. Mol Ther. 2011;19:620-6.

70. Pearce EL, Poffenberger MC, Chang CH, Jones RG. Fueling immunity: insights into metabolism and lymphocyte function. Science. 2013;342:6155-242.

71. Plitas G, Konopacki C, Wu K, Bos PD, Morrow M, Putintseva EV, Chudakov DM, Rudensky AY. Regulatory T cells exhibit distinct features in human breast cancer. Immunity. 2016;45(5):1122-34. 
72. Postow MA, et al. Immune checkpoint blockade in cancer therapy. J Clin Oncol. 2015;33:1974-82.

73. Restifo NP, Dudley ME, Rosenberg SA. Adoptive immunotherapy for cancer: harnessing the $\mathrm{T}$ cell response. Nat Rev Immunol. 2012;12:269-81.

74. Ribas A, Hodi FS, Callahan M, Konto C, Wolchok J. Hepatotoxicity with combination of vemurafenib and ipilimumab. N Engl J Med. 2013;368(14):1365-6.

75. Riemann D, Wenzel K, Schulz T, Hofmann S, Neef H, Lautenschläger C, Langner J. Phenotypic analysis of T lymphocytes isolated from non-small-cell lung cancer. Int Arch Allergy Immunol. 1997;114:38-45.

76. Rizvi N, Gettinger S, Goldman J. Safety and efficacy of firstlinenivolumab and ipilimumab in non-small cell lung cancer. In: 16th world conference on lung cancer, vol. 18; 2015. p. 6-9.

77. Robbins PF, Morgan RA, Feldman SA, Yang JC, Sherry RM, Dudley ME. Tumor regression in patients with metastatic synovial cell sarcoma and melanoma using genetically engineered lymphocytes reactive with NY-ESO-1. J Clin Oncol. 2011;29:917-24.

78. Robert C, Thomas L, Bondarenko I, O'Day S, Weber J, Garbe $\mathrm{C}$, et al. Ipilimumab plus dacarbazine for previously untreated metastatic melanoma. N Engl J Med. 2011;364(26):2517-26.

79. Rosenberg SA, Restifo NP, Yang JC, Morgan RA, Dudley ME. Adoptive cell transfer: a clinical path to effective cancer immunotherapy. Nat Rev Cancer. 2008;8:299-308.

80. Rosenberg SA, Dudley ME. Adoptive cell therapy for the treatment of patients with metastatic melanoma. Curr Opin Immunol. 2009;21:233-40.

81. Rains N, Cannan R, Chen W, Stubbs R. Development of a dendritic cell (DC) based vaccine for patients with advanced colorectal cancer. Hepatogastroenterology. 2000;43:184-203.

82. Rodriguez-Pinto D. B cells as antigen presenting cells. Cell Immunol. 2005;238:67-75.

83. Sampson JH, Vlahovic G, Sahebjam S, Omuro AMP, Baehring JM, Hafler DA. Preliminary safety and activity of nivolumab and its combination with ipilimumab in recurrent glioblastoma (GBM). J Clin Oncol. 2015;33:3010.

84. Schwartz M, Zhang Y, Rosenblatt JD. B cell regulation of the anti-tumor response and role in carcinogenesis. J Immunother Cancer. 2016;4:40-52

85. Sharma MD, Shinde R, McGaha T, Huang L, Holmgaard $\mathrm{RB}$, Wolchok JD. The PTEN pathway in Tregs is a critical driver of the suppressive tumor microenvironment. Sci Adv. 2015;1:150-84.

86. Shen C. Latex bead-based artificial antigen-presenting cells induce tumor-specific CTL responses in the native T-cell repertoires and inhibit tumor growth. Immunol Lett. 2013;150:1-11.

87. Sun S. Dual function of Drosophila cells as APCs for naive CD8+ T cells: implications for tumor immunotherapy. Immunity. 1996;4:555-64.

88. Shitara K, Nishikawa $\mathrm{H}$. Regulatory T cells: a potential target in cancer immunotherapy. Ann N Y Acad Sci. 2018;1417:104-15.

89. Sugiyama D, Nishikawa H, Maeda Y, Nishioka M, Tanemura A, Katayama I, Ezoe S, Kanakura Y, Sato E, Fukumori Y, Karbach J, Jäger E, Sakaguchi S. Anti-CCR89 mAb selectively depletes effector-type FoxP3+ CD4+ regulatory T cells, evoking antitumor immune responses in humans. Proc Natl Acad Sci USA. 2013;110(44):17945-50.

90. Tivol EA, Borriello F, Schweitzer AN, Lynch WP, Bluestone JA, Sharpe AH. Loss of CTLA-4 leads to massive lymphoproliferation and fatal multiorgan tissue destruction, revealing a critical negative regulatory role of CTLA-4. Immunity. 1995;3:541-7.

91. Topalian SL, Hodi FS, Brahmer JR, Gettinger SN, Smith DC, McDermott DF. Safety, activity, and immune correlates of antiPD-1 antibody in cancer. N Engl J Med. 2012;366:2443-54.
92. Trefzer U, Herberth G, Wohlan K, Milling A, Thiemann M, Sherev T, et al. Vaccination with hybrids of tumor and dendritic cells induces tumor-specific T-cell and clinical responses in melanoma stage III and IV patients. Int J Cancer. 2004;110:730-40.

93. Turtle CJ, Riddell SR. Artificial antigen-presenting cells for use in adoptive immunotherapy. Cancer J. 2010;16:374-81.

94. Ueno H, Schmitt N, Klechevsky E, Pedroza Gonzalez A, Matsui T, Zurawski G, Oh S, Fay J, Pascual V, Banchereau J, Palucka K. Harnessing human dendritic cell subsets for medicine. Immunol Rev. 2010;234(1):199-212.

95. Villarreal DO, L'Huillier A, Armington S, Mottershead C, Filippova EV, Coder BD, Petit RG, Princiotta MF. Targeting CCR95 induces protective antitumor immunity and enhances vaccine-induced responses in colon cancer. Cancer Res. 2018:78(18):5340-8.

96. Wahl DR, Byersdorfer CA, Ferrara JL, Opipari AW Jr, Glick GD. Distinct metabolic programs in activated T cells: opportunities for selective immunomodulation. Immunol Rev. 2012;249:104-15.

97. Ward E. A glycoengineered anti-CD19 antibody with potent antibody- dependent cellular cytotoxicity activity in vitro and lymphoma growth inhibition in vivo. $\mathrm{Br} \mathrm{J}$ Haematol. 2011;155:426-37.

98. Wang L, de Zoeten EF, Greene MI, Hancock WW. Immunomodulatory effects of deacetylase inhibitors: therapeutic targeting of FOXP3+ regulatory T cells. Nat Rev Drug Discov. 2009;8:969-81.

99. Ward ST, Li KK, Hepburn E, Weston CJ, Curbishley SM, Reynolds GM, et al. The effects of CCR99 inhibition on regulatory $\mathrm{T}$-cell recruitment to colorectal cancer. $\mathrm{Br} \mathrm{J}$ Cancer. 2015;112:319-28.

100. Wolchok JD, Kluger H, Callahan MK, Postow MA, Rizvi NA, Lesokhin AM, et al. Nivolumab plus ipilimumab in advanced melanoma. N Engl J Med. 2013;369(2):122-33.

101. Wshen P, Fillatreau S. Antibody-independentfunctions of B cells: a focus on cytokines. Nat Rev Immunol. 2015;15:441-51.

102. Xia J, Tanaka Y, Koido S, Liu C, Mukherjee P, Gendler SJ. Prevention of spontaneous breast carcinoma by prophylactic vaccination with dendritic/tumor fusion cells. J Immunol. 2003;170:1980-6.

103. Yan J, Mamula MJ. B and T cell tolerance and autoimmunity in autoantibody transgenic mice. Int Immunol. 2002;14:963-71.

104. Yoon JT, Longtine MS, Marquez-Nostra BV, Wahl RL. Evaluation of next-generation anti-CD20 antibodies labeled with zirconium 89 in human lymphoma xenografts. J Nucl Med. 2018;58:49-52.

105. Zeng $\mathrm{H}$, Chi $\mathrm{H}$. The interplay between regulatory $\mathrm{T}$ cells and metabolism in immune regulation. Oncoimmunology. 2013;2:11-86.

106. Zhang Z, Zhu Y, Wang Z, et al. Yin-yang effect of tumor infiltrating B cells in breast cancer: from mechanism to immunotherapy. Cancer Lett. 2017;393:1-7.

107. Zheng Y, Josefowicz SZ, Kas A, Chu TT, Gavin MA, Rudensky AY. Genome-wide analysis of Foxp3 targetgenes in developing and mature regulatory T cells. Nature. 2007;445:936-40.

108. Zuang H, Benoist C, Mathis D. Rituximab specifically depletes short-lived autoreactive plasma cells in a mouse model of inflammatory arthritis. Proc Natl Acad Sci USA. 2010;107:4658-63.

Publisher's Note Springer Nature remains neutral with regard to jurisdictional claims in published maps and institutional affiliations. 